\title{
O método lógico-histórico nas análises epistemológicas: a experiência brasileira no campo da educação física
}

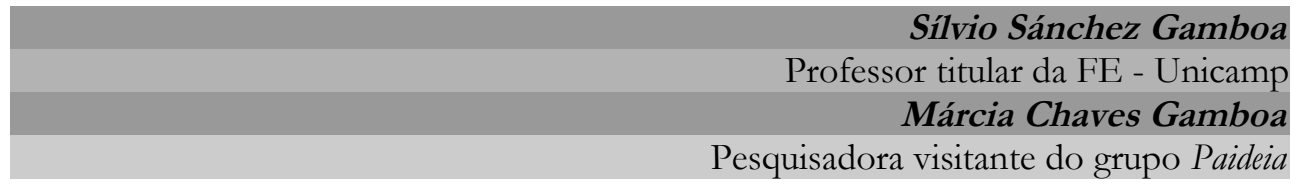

\section{Resumo}

O método lógico-histórico é uma ferramenta de análise epistemológica da produção científica que ajuda, em primeiro lugar, a recuperar suas características, sua lógica interna, o modelo paradigmático ou epistemologia dominante nas teses, ou relatórios de pesquisa. Em segundo lugar, ajuda a recuperar as condições materiais, institucionais e políticas que determinam a produção e revelam sua inserção em perspectivas, tendências, concepções científicas privilegiadas e a influência de políticas científicas, interesses e ideologias sociais dominantes. Os resultados indicam que a formação do campo científico da Educação Física ainda está na fase da "guerra da ciência". Confirma-se assim, que a produção científica não é neutra e que obedece a interesses dominantes nas regiões, instituições, grupos de pesquisa e estruturas sociais.

Palavras-chave: Análise epistemológica; Pesquisa educacional; Método lógicohistórico.

\section{Resumen}

El método lógico-histórico es una herramienta del análisis epistemológico de la producción científica que ayuda, en primer lugar, a recuperar las características, su lógica interna, el modelo paradigmático o, la epistemología dominante en las tesis, o informes de investigación. En segundo lugar, ayuda a recuperar las condiciones materiales, institucionales y políticas que determinan esa producción y revelan su inserción en perspectivas, tendencias, concepciones científicas privilegiadas y la influencia de políticas de científicas, intereses e ideologías sociales dominantes. Os resultados indican que la constitución del campo científico de la Educación Física aún está en la fase de la "guerra de las ciencias". Confirma-se así, que la producción científica no es axiológicamente neutra y que obedece a intereses dominantes en las regiones, las instituciones, en los grupos de investigación y en las estructuras sociales.

Palabras clave: Análisis epistemológico; Investigación educativa; Método lógico-histórico.

Filosofia e Educação (Online), ISSN 1984-9605 - Volume 6, Número 2, Junho de 2014 
Introdução

$\mathrm{E}$

ste trabalho apresenta resultados relativos à problemática da constituição do campo científico da área da Educação Física com base no projeto temático: "Produção do conhecimento em educação física: impacto do sistema de pós-graduação das regiões sul e sudeste do Brasil na formação e produção de mestres e doutores que atuam nas instituições de ensino superior da região nordeste", financiado pela Fundação de Amparo à pesquisa do estado de São Paulo - FAPESP (proc. 2012/50019-7). Os resultados relativos à constituição do campo científico indicam pontos em conflitos relacionados com: a) a rivalidade entre as ciências naturais (Física, Anatomia, Biomecânica, Fisiologia, Física) e as ciências humanas y sociais (Pedagogia, Sociologia, Psicologia, Historia, Filosofia) nos currículos de formação profissional b) as controvérsias entre métodos quantitativos e qualitativos nos projetos de pesquisa; c) a busca da objetividade nas pesquisas analíticas ou da explicitação da subjetividade nas pesquisas interpretativas. Esses conflitos desafiam aos cientistas que analisam a produção do conhecimento. Os estudos meta científicos realizados sobre a produção da educação física do nordeste brasileiro sinalizam que esses conflitos podem ser esclarecidos e compreendidos, quando a produção científica é interpretada sob a perspectiva da epistemologia dialética e dos fundamentos do método lógico-histórico. O trabalho foi organizado em três partes: 1) Conflitos e polarizações na constituição do campo científico da Educação Física; 2) estudos meta científicos e o método lógico histórico; 3) Perspectivas de superação dos conflitos e das polarizações na formação do campo científico na Educação Física. Algumas conclusões frisam a necessidade de uma vigilância epistemológica sobre a produção do conhecimento como uma forma de superar os conflitos e as polarizações no campo da formação profissional e no campo científico da área.

Filosofia e Educação (Online), ISSN 1984-9605 - Volume 6, Número 2, Junho de 2014 
1. Conflitos e polarizações na constituição do campo científico da Educação Física

Considerando o conceito de campo de Bourdieu (1983), a Educação Física recentemente no Brasil passa pela transformação do campo profissional, relacionado com a formação e o reconhecimento oficial da profissão para um novo campo de estudos e pesquisas sobre a prática profissional, constituindo assim seu campo científico. Um marco de referência dessa transformação está no surgimento de projetos de pesquisa que ganha força e estatuto científico na criação e consolidação dos programas de pósgraduação $(1977)^{1}$. O campo científico é um campo de conflitos teóricometodológicos. Para caracterizar esses conflitos recorremos às análises epistemológicas dos currículos de formação profissional e à análise da produção do conhecimento na área, tendo como fonte a produção dos programas de pós-graduação. Essa produção é analisada com base nas tendências teórico-metodológicas que ajudam a diferenciar diversas formas ou modelos de produção do conhecimento científico e critérios diferenciados de validar esse conhecimento e justificar formas diversas de atuação profissional. A análise das tendências teórico-metodológicas, ou dos paradigmas científicos que permeiam as análises, ou dos critérios da atuação profissional supõe a articulação entre os níveis técnico-instrumental, metodológico e teórico, além de abrir a possibilidade de identificar pressupostos filosóficos que permitem a identificação de perspectivas que, na forma de pressupostos, relacionam a prática da pesquisa e a prática profissional com visões de mundo e os interesses humanos.

Os resultados relativos à constituição do campo científico indicam pontos em conflitos e de polarizações relacionados com: a) a rivalidade entre as ciências naturais (Física, Anatomia, Biomecânica, Fisiologia, Física) e as ciências humanas y sociais (Pedagogia, Sociologia, Psicologia, Historia, Filosofia) nos currículos de formação profissional b) as controvérsias entre métodos quantitativos e qualitativos nos projetos de

${ }^{1} \mathrm{O} 1^{\circ}$ programa de pós-graduação foi criado na Universidade de São Paulo - USP. Por ocasião do início da pesquisa temática (2012) existiam 23 cursos recomendados pela Coordenação de Aperfeiçoamento do Pessoal do Ensino Superior - CAPES.

Filosofia e Educação (Online), ISSN 1984-9605 - Volume 6, Número 2, Junho de 2014 
pesquisa e c) a busca da objetividade nas pesquisas analíticas ou da explicitação da subjetividade nas pesquisas interpretativas.

Com relação às rivalidades entre ciências naturais e ciências humanas e sociais, o histórico da pós-graduação na área já anuncia esse conflito. A primeira experiência de pós-graduação stricto sensu na Educação Física brasileira é de 1977 na USP, que até o final daquela década se colocava como referência hegemônica na formação dos pesquisadores profissionais da área, centrada no aporte biofisiológico da aptidão física, entretanto, sem considerar sua natureza histórico-social. De lá para cá se assistiu ao processo de construção e desenvolvimento da Educação Física em sua estrutura acadêmica espelhada na ainda hegemônica - mesmo que ao lado de movimentos contra hegemônicos significativos - da presença do paradigma biológico na configuração de sua compreensão de ciência, de pesquisa e de formação do pesquisador. Tal quadro explica a inserção dos programas de Educação Física no contexto da política de pós-graduação brasileira na grande área da saúde, estabelecendo critérios de excelência acadêmica com os programas de fisioterapia, terapia ocupacional, enfermagem e odontologia. Em defesa dessa localização Kokubun (2003 e 2004) polemiza com Lovisolo (1998), Betti, Carvalho, Daólio e Pires (2004) num debate que contrapõe pesquisas que se valem dos conhecimentos oriundos das ciências naturais (biodinâmica) àquelas que buscam seu alicerce nas ciências sociais e humanidades (socioculturais), diferenciando os critérios de avaliação utilizados na grande área de Saúde dos utilizados na subárea sociocultural. Os autores partilham o critério da avaliação da pós-graduação na grande área da Saúde, mas discordam sobre a necessidade de um trato especial da subárea sociocultural e sobre a manutenção dos instrumentos e concepção de ciência atualmente predominante na avaliação da CAPES ou sobre a necessidade de agregar indicadores de natureza qualitativa e a superação do modelo tradicional de ciência, baseado na superespecialização, hierarquia de áreas e acumulação de conhecimentos.

Com relação à polêmica entre métodos quantitativos e qualitativos é uma consequência da anterior já as concepções de ciência predominante na área das biológicas são privilegiados métodos empírico-analíticos que, no

Filosofia e Educação (Online), ISSN 1984-9605 - Volume 6, Número 2, Junho de 2014 
contexto brasileiro, frisam a necessidade da quantificação das variáveis que compõem os desenhos experimentais e quase experimentais e organizam a pesquisa sob a perspectiva do raciocínio hipotético-dedutivo. A presença das perspectivas das ciências humanas é associada aos métodos compreensivos e históricos. Na análise da produção da pós-graduação são identificados, dentre os paradigmas dominantes na Educação Física, em primeiro lugar, os modelos de pesquisa desenvolvidos na física, a mecânica, na biologia e na psicologia fundamentados numa concepção empíricoanalítica de ciência. Posteriormente com a influência da sociologia da antropologia e as ciências da educação surgem alternativas científicas, identificadas como os paradigmas compreensivos e históricos (fenomenologia e dialética) e recentemente com outros paradigmas emergentes (SANTOS, 1996).

A busca da objetividade nas pesquisas analíticas ou da explicitação da subjetividade nas pesquisas compreensivas e interpretativas se apresenta também no universo da produção da pós-graduação. A justificação da oposição ou incompatibilidade entre as duas abordagens, se sustenta no argumento da especificidade dos objetos que são estudados: a) as ciências naturais, tais como a física, a química, a biologia e a mecânica precisam preservar a objetividade e utilizar uma linguagem que filtre as apreciações subjetivas e a forma mais segura é a utilização da linguagem matemática e estatística, já as ciências sociais e humanas, carregadas de subjetividades, devem privilegiar a interpretação e as linguagens polissêmicas, difíceis de serem reduzidas a códigos matemáticos.

\section{Os estudos metacientíficos e o método lógico-histórico}

Os estudos metacientíficos analisam a produção científica, buscando sua caracterização com relação a sua configuração teórica e metodológica, e contribuindo para apontar focos, núcleos temáticos, referenciais teóricos, meios, recursos e processos adotados na produção do conhecimento.

A pesquisa meta-analítica da produção científica caracteriza-se como um tipo de pesquisa-avaliação. As pesquisas meta-analíticas são importantes, principalmente para os programas de pós-graduação, já que

Filosofia e Educação (Online), ISSN 1984-9605 - Volume 6, Número 2, Junho de 2014 
permitem aos interessados (docentes, estudantes, pesquisadores, universidades, agências de fomento) apreciar criticamente o conhecimento produzido, detectar entraves e investir da melhor forma na produção, tanto em termos de produtos como de processos (LAROCCA; ROSSO; SOUZA, 2005, p.119).

Um dos estudos metacientíficos mais desenvolvidos se referem às análises epistemológicas que se constituem num um campo intermediário entre as práticas científicas e a reflexão filosófica, razão pela qual se conhece também pelos sinônimos de Teoria da ciência, Metaciência ou Filosofia da Ciência (BLANCHÉ, 1975; BACHELARD, 1989). O conceito de epistemologia tem a sua origem na composição grega episteme (conhecimento) e logos, (razão, explicação), e significa o estudo da natureza do conhecimento, a sua justificação e seus limites (AUDI, 2004). Essas três dimensões são representadas pelas controvérsias filosóficas acerca da possibilidade, das fontes, da essência e dos critérios de validade de um conhecimento sistemático (episteme), o qual, por ter o imperativo de explicitar e justificar os métodos ou os caminhos e formas da elaboração dos seus resultados, que se diferencia historicamente de outras formas de encontrar, tais como o senso comum (Doxa) e a razão mítica (Mitus) (SÁNCHEZ GAMBOA, 2005).

A epistemologia contemporânea vem se reconstruindo na interface entre a ciência e a filosofia. Segundo Habermas (1982), a filosofia moderna e particularmente Kant propiciou organizou o conhecimento acumulado em dois grandes campos, propondo a fronteira entre a "Razão prática" e a "Razão pura", propiciando dessa forma a ruptura entre a Filosofia e a Ciência. Tal ruptura vem sendo superada pela filosofia contemporânea e vem acontecendo "crítica e reflexivamente" na epistemologia, entendida esta como o estudo sistemático que encontra na Filosofia seus princípios e na produção científica seu objeto. Nesse sentido, “A filosofia preserva-se na ciência enquanto crítica" (HABERMAS, 1982, p. 77).

Dentre as diversas formas de desenvolvimento das epistemologias contemporâneas, a dialética, caracterizada como "Lógica e Teoria do Conhecimento" (KOPNIN, 1978), apresenta importantes ferramentas para

Filosofia e Educação (Online), ISSN 1984-9605 - Volume 6, Número 2, Junho de 2014 
desenvolver essa reflexão crítica sobre a produção do conhecimento científico. Tal perspectiva epistemológica oferece recursos para reconstituir a unidade na análise da ciência em seus aspectos internos (lógicos, gnosiológicos e metodológicos) e externos (histórico-sociais). Nesse sentido, a dialética desenvolve a ideia da unidade entre Epistemologia e a Teoria do Conhecimento e as condições materiais e históricas da produção do conhecimento. A base materialista e histórica da lógica dialética permite superar a separação, proposta pelo idealismo alemão, entre ontologia (que trata da realidade) e a gnosiologia (que trata do conhecimento dessa realidade). Superação que acontece quando a realidade e o seu conhecimento são concretos e determinados pelas condições concretas da produção desse conhecimento.

A análise epistemológica na perspectiva da dialética supõe a compreensão da obra científica como um todo lógico que articula diversos fatores, os quais lhe dão unidade de sentido. Essa unidade de sentido se produz em condições histórico-sociais que determinam e caracterizam a obra científica como única e como parte do processo maior da produção do conhecimento humano. Assim, as categorias centrais da análise referem-se à relação entre o lógico e o histórico. Em outras palavras, o trabalho científico (neste caso, a pesquisa em Educação Física) abrange conteúdos lógicos e históricos. $\mathrm{O}$ conteúdo lógico se refere à articulação de noções e categorias que formam uma unidade de pensamento ou uma estrutura interna mais ou menos formalizada. Tal unidade não se apresenta fixa ou terminada, pois obedece a um processo de produção e de gênese, razão pela qual o lógico não pode estar separado do histórico.

No caso concreto da análise epistemológica da produção científica em educação Física a inter-relação entre o lógico e o histórico se refere a dois momentos específicos. No primeiro procura-se a estrutura interna implícita em toda pesquisa, a forma como define e articula seus elementos constitutivos; no segundo recuperam-se as condições históricas que determinam essa produção. A integração destes dois momentos que se explicitam mutuamente nos oferece um conhecimento sobre as características não apenas de cada pesquisa em particular, mas do

Filosofia e Educação (Online), ISSN 1984-9605 - Volume 6, Número 2, Junho de 2014 
movimento do pensamento que a sustenta e da tendência científica na qual se situa. $\mathrm{O}$ estudo lógico pretende reconstituir os modelos epistemológicos predominantes nas pesquisas, caracterizando-as de acordo com os níveis de articulação explícitos (níveis técnico-metodológico, teórico e epistemológico) e de pressupostos implícitos (gnosiológicos e ontológicos). O estudo histórico busca elucidar as tendências desses modelos ou paradigmas epistemológicos, recuperar as condições históricas, sociais, políticas, administrativas e acadêmicas que explicam a trajetória dessas tendências epistemológicas.

A caracterização dos pressupostos epistemológicos, tanto nos aspectos internos (lógicos) como externos (históricos) de uma obra científica ajuda a diferenciar diversas formas ou modelos de produção do conhecimento científico e critérios diferenciados de validar esse conhecimento, formas que são também conhecidas como paradigmas científicos ou matrizes epistemológicas. Essas matrizes podem ser caracterizadas, particularmente na análise da produção acadêmica (teses, dissertações, relatórios de pesquisa, monografias).

Esse exercício de recuperação da lógica que caracteriza as pesquisas ganham mais sentido e validade quando se recuperam também as dimensões históricas da produção da pesquisa, nas condições concretas do Nordeste brasileiro.

Tal preocupação metodológica que articula o lógico e o histórico possibilita a compreensão das contradições entre as categorias ou teorias utilizadas para caracterizar e classificar a produção e a prática concreta da produção do conhecimento (KOSIK, 1976). A relação entre o lógico e histórico obriga a reconsiderar as matrizes conceituais e a caracterização das pesquisas à luz dessas matrizes quando se recuperam os determinantes históricos da produção da pesquisa científica atrelada aos programas de pósgraduação concentrados na região Sudeste e determinados por uma política de ciência e tecnologia que aprofunda as desigualdades regionais.

Filosofia e Educação (Online), ISSN 1984-9605 - Volume 6, Número 2, Junho de 2014 
3. Perspectivas de superação dos conflitos e as polarizações na formação do campo científico na Educação Física

Com base nas análises epistemológicas Chaves (2005) elaborou um balanço crítico da produção de mestres e doutores que atuam nos estados nordestinos de Alagoas, Bahia, Pernambuco e Sergipe com o objetivo de identificar tendências, perspectivas e desafios para a consolidação da política de pósgraduação e pesquisa científica em Educação Física no Nordeste do Brasil. O estudo registrou 145 pesquisas distribuídas nos estados de Pernambuco (62); Bahia (35); Alagoas (23); e Sergipe (25), entretanto, os registros de catalogação foram limitados a 122 pesquisas defendidas: 96 dissertações e 26 teses. Desse total foram analisadas 70 pesquisas (58 dissertações, 11 teses de doutorado e 01 tese de pós-doutorado), produzidas entre 1982 e 2004. Com base no método lógico, instrumentalizado na matriz epistemologia proposta por Sánchez Gamboa (1987) foi possível obter resultados que identificam as áreas pesquisadas: memória, cultura e corpo (21\%), educação física escolar (20\%), formação profissional/campo de trabalho (19\%), epistemologia (09\%), políticas públicas (09\%), atividade física e saúde $(07 \%)$, recreação/lazer $(06 \%)$, portadores de necessidades especiais (04\%), movimentos sociais (04\%) e rendimento de alto nível $(01 \%)^{2}$. Com relação ao enfoque teórico-metodológico, constatou-se a expansão da apropriação da abordagem crítico-dialética (46\%) e fenomenológica-hermenêutica (34\%), com redução do enfoque empíricoanalítica $(16 \%)$. Cabe pontuar que esta é uma tendência presente no Nordeste que se diferencia de outros resultados sobre a produção nacional de dissertações e teses apresentados por Silva (1998 e 1990) que identificou o predomínio do enfoque empírico analítico.

O método histórico ajudou a recuperar as condições da produção no período compreendido entre 1982 e 2004, abrangendo 22 anos. Considerando a primeira dissertação defendida (1982), a primeira tese

\footnotetext{
${ }^{2}$ A classificação temática corresponde à utilizada pelo Colégio Brasileiro de Ciências do Esporte - CBCE - para a distribuição dos Grupos de Trabalho Temático (GTT) que aglutinam interesses de estudos e pesquisas na área da Educação Física. Os critérios para a criação desses GTTs dependem de interesses de pesquisadores e do volume comprovado da produção sobre essa temática. Tais critérios foram considerados nas classificações temáticas utilizadas nas pesquisas sobre a produção no nordeste.
}

Filosofia e Educação (Online), ISSN 1984-9605 - Volume 6, Número 2, Junho de 2014 
produzida na região (1993) e a consolidação de redes de intercâmbio e de grupos de pesquisa (2000), a produção foi classificada em três períodos: pioneirismo (1982-1992), expansão (1993-1999) e consolidação (20002004). A estruturação desta periodização permitiu visualizar as tendências temáticas e epistemológicas dominantes em cada período e sua evolução nos 22 anos de produção.

Os resultados limitados a quatro estados do nordeste suscitou a elaboração de um novo projeto que abrange os noves estados (Alagoas, Bahia, Pernambuco e Sergipe, Ceará, Maranhão, Paraíba, Piauí e Rio Grande do Norte) e sua ampliação com relação às influências teóricas que determinam a formação dos pesquisadores que atuam nos 123 cursos de educação física, localizados nas 467 Instituições de Ensino Superior - IES da região. O estudo também caracteriza os impactos dos programas de pósgraduação das regiões Sul e Sudeste na formação dos pesquisadores que atuam Nordeste, particularmente a influência do Sudeste, onde se concentram 54\% dos programas de pós-graduação em Educação Física do país e são responsáveis por $62.6 \%$ das dissertações e $92.5 \%$ das teses, especificamente, os programas localizados no estado de São Paulo registram $47.1 \%$ das dissertações e $72.1 \%$ das teses produzidas no país. Esses indicadores evidenciam a distribuição desigual e a concentração da produção no sudeste e a necessidade de mudanças nas políticas de desenvolvimento da ciência e tecnologia.

Além de registrar as desigualdades regionais e revelar os conflitos das políticas de pós-graduação, os estudos epistemológicos também revelam um significativo número de pesquisas vinculada às ciências humanas, particularmente à educação $(51 \%)$, a outras áreas $(0,6 \%)^{3}$, e a programas em Educação Física, em outras regiões do Brasil ou fora do país (43\%). O predomínio de pesquisas defendidas em outros programas, além dos específicos da área da Educação Física, levanta a seguinte hipótese:

\footnotetext{
3 Além da Educação as outras áreas são: Serviço Social e Sociologia. Das pesquisas defendidas em programas de pós-graduação, sediados no nordeste, $65 \%$ são da área da Educação.
}

Filosofia e Educação (Online), ISSN 1984-9605 - Volume 6, Número 2, Junho de 2014 
A formação do campo científico da Educação Física, depende das inter-relações necessárias com outros campos do conhecimento, tais como a educação, as ciências sociais e as artes, como confirmam as áreas de formação dos pesquisadores e o desenvolvimento interdisciplinar das suas teses e dissertações" (CHAVES-GAMBOA; SÁNCHEZ GAMBOA 2009, p. 142).

O número significativo de pesquisas defendidas em programas vinculados às ciências humanas indicam a consolidação das abordagens interdisciplinares e a superação da bipolaridade Ciências Biológicas/Humanidades com o subsequente fortalecimento dos campos acadêmicos no processo de constituição do campo profisssional e científico da Educação Física.

Em relação às categorias quantidade/qualidade, o método lógico histórico também ajuda a compreender a síntese, ou unidade entre elas, defendida pela dialética marxista, que procura entender o conflito como a unidade de contrários. A perspectiva da unidade denuncia o falso "dualismo técnico" e a falsa "opção epistemológica" entre positivismo (quantidade) e a fenomenologia (qualidade) e anuncia os "terceiros excluídos": a dialética (a unidade de opostos: quantidade e qualidade). O conflito entre métodos explicativos (Durkheim) e compreensivos (Weber) podem ser superados na síntese de opostos (Marx) (Cfr. Löwy, 1986).

A unidade quantidade qualidade, segundo Sánchez Gamboa (2013) se expressa na unidade ontológica dos fenômenos que abrange diversas dimensões, entre elas, as quantitativas e qualitativas. A unidade entre as categorias quantidade/qualidade se revelam particularmente nas pesquisas com enfoque dialético, que utilizam técnicas historiográficas para recuperar os processos de transformação dos fenômenos. No processo de transformação as dimensões quantitativas e qualitativas modificam-se, complementam-se e transformam-se uma na outra, e vice-versa, quando aplicadas a um mesmo fenômeno. "As duas dimensões se inter-relacionam como duas fases do real num movimento cumulativo e transformador, de tal maneira que não podemos concebê-las uma sem a outra, nem uma separada da outra" (Sánchez Gamboa, 2013, p. 105).

Filosofia e Educação (Online), ISSN 1984-9605 - Volume 6, Número 2, Junho de 2014 


\section{Conclusões}

Os resultados das pesquisas sobre a produção do conhecimento no nordeste brasileiro indicam que a constituição do campo científico da Educação Física vem superando as dicotomias científicas e as polarizações entre ciências naturais e sociais. O método lógico histórico revela determinações e afiliações teóricas que obedecem a interesses dominantes nas regiões, instituições, áreas de conhecimento e estruturas sociais, mas, também revelam processos de superação e transformação na constituição dos campos profissional e científico.

O método lógico-histórico é uma ferramenta de análise epistemológica que ajuda, a recuperar as características, ou a lógica interna, ou epistemologia dominante nas teses e dissertações e a recuperar as condições materiais, institucionais e políticas que determinam essa produção e revela sua inserção nas perspectivas, e tendências dominantes nos campos profissional e científico, mas ao mesmo tempo, oferece recursos para revelar os processos de transformação e superação.

A compreensão das lógicas construídas nas dissertações e teses determinadas pelas condições materiais históricas dos programas de pósgraduação, assim como pelas políticas de ciência e tecnologia e as influências do sistema de pós-graduação nacional, centralizado na região Sudeste na produção do Nordeste, revelam a necessidade de mudança na organização do sistema de pós-graduação, superando as desigualdades regionais e a bipolaridade Ciências Biológicas/Humanidades que dificultam o desenvolvimento do campo científico da área no país.

\section{Referências}

AUDI, R. Dicionário AKAL de Filosofia. Madrid: AKAL, 2004.

BACHELARD, G. Epistemologia. Barcelona: Anagrama, 1989.

BETTI, M.; CARVALHO, Y. M.; DAÓLIO, J.; PIRES, G. D. L. A avaliação da pós-graduação em Educação Física em debate: implicações para a subárea pedagógica e sociocultural. Revista Brasileira de Pós-Graduação - R B P G, v. 1, n. 2, p. 183-194, nov. 2004.

BOURDIEU, P. O campo científico. In: ORTIZ, R. (org.). Pierre Bourdieu: sociologia. São Paulo: Ática, 1983.

Filosofia e Educação (Online), ISSN 1984-9605 - Volume 6, Número 2, Junho de 2014 
CHAVES, M. A produção do conbecimento em Educação Física nos Estados do Nordeste (Alagoas, Babia, Pernambuco e Sergipe) 1982-2004: balanço e perspectivas. 2005. Tese (Pós-doutorado em Educação), Faculdade de Educação, Universidade Federal da Bahia, Salvador, 2005.

CHAVES-GAMBOA, M.; SÁNCHEZ GAMBOA, S. Pesquisa na Educação Física: epistemologias, escola e formação profissional. Maceió: edUFAL, 2009

KOKUBUN, E. Pós-graduação em educação física no Brasil: indicadores objetivos dos desafios e das perspectivas. Revista Brasileira de Ciências do Esporte, v. 24, n. 2, p. 9-26, 2003.

KOKUBUN. E. A avaliação da Educação Física em debate: esclarecimentos, Revista Brasileira de Pós-Graduaşão - R B P G , v. 1, n. 2, p. 195-200, nov. 2004.

KOPNIN, P. V. A dialética como lógica e teoria do conhecimento. Rio de Janeiro: Civilização Brasileira, 1978.

KOSIK. K. Dialética do concreto. Rio de Janeiro: Paz e Terra, 1976.

LAROCCA. P.; ROSSO, A J. SOUZA A P. A formulação dos objetivos de pesquisa na pós-graduação em Educação: uma discussão necessária. Brasília: R B P G, v. 2, n. 3, p. 118-133, mar. 2005.

LOVISOLO, H. Pós-graduações e educação física: paradoxos, tensões e diálogos. Revista Brasileira de Ciências do Esporte, v. 20, n. 1, p. 11-21, 1998.

LÖWY, M. Ideologia e ciências sociais. São Paulo: Cortez, 1986.

SÁNCHEZ GAMBOA. S. Epistemologia da Educação Física: as inter-relações necessárias. 2.ed. Maceió: edUFAL, 2010.

SÁNCHEZ GAMBOA. S. Epistemologia da pesquisa em educação: estruturas lógicas e tendências metodológicas. 1987. Tese (Doutorado em Educação) - Faculdade de Educação, Universidade Estadual de Campinas, Campinas, 1987.

SÁNCHEZ GAMBOA. S. Pesquisa em Educação: métodos e epistemologias. Chapecó, SC: Argos, 2012.

SÁNCHEZ GAMBOA. S.; SANTOS FILHO, C. (Org.). Pesquisa educacional: qualidade-quantidade. 8.ed. São Paulo: Cortez, 2013.

SANTOS, B de S. Um discurso sobre as ciências. Porto: Afrontamento, 1996.

SILVA, R. V. S. Mestrados em Educação Física no Brasil: pesquisando a suas pesquisas. 1990. Dissertação (Mestrado) - Educação Física, Universidade de Santa Maria, Santa Maria, 1990.

SILVA, R. V. S. Pesquisa em Educação Física: determinações históricas e implicações metodológicas. 1997. Tese (Doutorado em Educação) - Faculdade de Educação, Universidade Estadual de Campinas, Campinas, 1997.

Filosofia e Educação (Online), ISSN 1984-9605 - Volume 6, Número 2, Junho de 2014 